\title{
Treatment of phytobezoars: Tailoring management to diverse presentations
}

Zeki Özsoy (), İsmail Okan (1)

ABSTRACT Objective: A minimally invasive approach is feasible and successful for the treatment of phytobezoars. We would like to draw attention to the overconsumption of Sorbus domestica and discuss the management options of patients presenting with different symptoms due to phytobezoars.

Material and Methods: Data from patients diagnosed with phytobezoars in the Department of General Surgery from 2010 to 2016 were prospectively collected and evaluated.

Results: Twenty patients diagnosed with phytobezoars were included in the study. The etiology of phytobezoar was Sorbus domestica seeds in 12 patients, watermelon seeds in two patients, Japanese persimmon seeds in one patient, and unidentified in five patients. Fourteen patients underwent surgery, while the remaining patients were treated conservatively. Minimally invasive surgery was used in all but two cases, where the surgery was converted to laparotomy. Two patients developed postoperative morbidity. There was no mortality.

Conclusion: The treatment modality should be selected for each patient according to the presenting symptoms and characteristics of phytobezoar. In cases where surgery is performed, a minimally invasive approach is feasible and successful for the treatment of ileus.

Keywords: Minimally invasive approach, phytobezoar, sorbus domestica, treatment

ORCID IDs of the authors: Z.0.0000-0003-1305-5767, I. $0.0000-0001-8110-356 \mathrm{X}$

\section{Cite this paper as:} Özsoy Z, Okan I. Treatment of phytobezoars: Tailoring management to diverse presentations. Turk J Surg 2018; 34: 33-37.

Department of General Surgery, Gaziosmanpaşa University School of Medicine, Tokat, Turkey

Address for Correspondence Zeki Özsoy

e-mail:zekiserkanozsoy@hotmail.com

Received: 19.11.2016

Accepted: 03.03.2017

Available Online Date: 04.01.2018

CCopyright 2018

by Turkish Surgical Association

Available online at

www.turkjsurg.com

\section{INTRODUCTION}

Bezoars can be defined as the accumulation of indigestible materials, such as hair, vegetable and fruit seeds, fibers, and skin, in the gastrointestinal system. Phytobezoars are accumulations of indigestible fibers and seeds of fruits and vegetables; trichobezoars comprise hair, bristles, and feathers; lactobezoars are formed of milk and milk products; and pharmacobezoars are the result of copious intake of medication. Phytobezoars are the most common form of bezoar and are usually detected in the stomach (1). The presentation of bezoars may vary from subtle, dyspepsia-like symptoms to more pronounced findings, such as ileus. However, in rare cases of prolonged exposure, bezoars can induce serious inflammatory changes, leading to ulcer formation, perforation, and misdiagnosis of tumor.

Phytobezoars are mostly encountered in patients with digestive and gastric motility problems, diabetes mellitus (DM) with gastropathy, and gastric resections. Seeds of fiber-rich fruits and vegetables and indigestible material can accumulate in any part of the gastrointestinal system where the passage is slowed or obstructed. Many fruit and vegetable materials, such as banana, persimmon, coconut, Brussels sprout, and potato peel, have been reported to cause phytobezoars and subsequent ileus (2). The use of herbs, fruits, and vegetables for the treatment of bezoars is common in folk medicine (3). Sorbus domestica (SD) has been traditionally used for the treatment of DM in Turkey and other parts of Europe (4). However, there has been no report in the literature of ileus due to phytobezoar formation caused by SD (Figure 1). In this study, we seek to draw attention to the issue of overconsumption of SD (called üvez in Turkey) and discuss management options for patients presenting with different symptoms.

\section{MATERIAL AND METHODS}

Data on patients diagnosed with phytobezoar in the Gaziosmanpaşa University School of Medicine, Department of General Surgery from 2010 to 2016 were collected. Because this was a retrospective study, we did not apply for ethical committee approval. This study was performed in accordance with the Declaration of Helsinki. The patients were diagnosed through esophagogastroduodenoscopy (EGD), abdominal computed tomography (CT), or admission for surgery due to ileus. When phytobezoar was suspected or diagnosed, a careful history of diet, concomitant diseases, previous surgery, and drugs used was taken, and a thorough physical examination was performed. If the patient presented with ileus, routine diagnostic (plain abdominal X-ray, abdominal CT, etc.) and treatment modalities (intravenous hydration, patient monitoring, etc.) were employed. In cases of surgery, patients underwent minimally invasive operations. Written informed consent was obtained from patients participated in this study. The demographic data, dietary habits, presenting symptoms and findings, laboratory values, 
operations, postoperative complications, and follow-up data of the patients were prospectively collected and evaluated.

\section{Statistical Analysis}

For statistical evaluation, Statistical Package for the Social Sciences version 15.0 (SPSS Inc.; Chicago, IL, USA) was used. For descriptive data, numeric values were given as mean \pm standard deviation and categoric values were given as rates.

Table 1. Demographic and clinical characteristics of the patients

\begin{tabular}{|lcc|} 
& $\mathbf{n}$ & $\%$ \\
\hline Patients & 20 & \\
\hline Gender & & \\
Male & 11 & 55 \\
Female & 9 & 45 \\
\hline Age (years, mean \pm standard deviation) & $56.7 \pm 14.3$ & \\
\hline
\end{tabular}

\section{Presenting symptoms and findings}

Dyspepsia, nausea, and vomiting

lleus

14

70

$\begin{array}{lcc}\text { Comorbidities } & & \\ \text { DM } & 7 & 35 \\ \text { CRF } & 1 & 5 \\ \text { None } & 12 & 60\end{array}$

Previous gastric surgery

$\begin{array}{lcc}\text { Yes } & 3 & 15 \\ \text { No } & 17 & 85\end{array}$

$\begin{array}{lcc}\text { Etiology } & & \\ \text { Sorbus domestica } & 12 & 60 \\ \text { Watermelon seeds } & 210 & \\ \text { Japanese persimmon } & 1 & 5 \\ \text { Unidentified } & 5 & 25\end{array}$

Time between ingestion and $2.75 \pm 1.25$ presentation of symptoms (months, mean \pm standard deviation)

DM: diabetes mellitus; CRF: chronic renal failure

\section{RESULTS}

Twenty patients with a diagnosis of phytobezoar were included in this study. The mean age of the patients was $56.7 \pm 14.3$ years, and 11 patients were male. Fourteen patients presented to the emergency department with ileus; phytobezoar was diagnosed via abdominal CT. Six patients presented with dyspepsia, nausea, and vomiting; phytobezoar was diagnosed with EGD and abdominal CT. Two patients were diagnosed during surgery. The etiologies of phytobezoar were SD seeds in 12 patients, watermelon seeds in two patients, Japanese persimmon seeds in one patient, and unidentified in five patients. The demographic and clinical details of the patients are presented in Table 1. Fourteen patients underwent surgery, while the remaining patients were treated conservatively. The details of clinical management are presented in Table 2.

Minimally invasive surgery was used in all but two cases, where the surgery was converted to laparotomy. In one patient, we found a phytobezoar in the descending colon during surgery. Because it was too dangerous to propagate the bolus using laparoscopy due to its spiked edges, the contents were crushed and passed through the anal canal via laparotomy. A second patient presented with acute mechanical intestinal obstruction; she was monitored for three days. In abdominal CT, an obstructing mass was identified in the terminal ileum. During laparotomy, an obstructing ileal mass causing partial necrosis and edema in the intestinal wall was detected. Segmental ileal resection and end-to-end intestinal anastomosis were performed. Three more bezoars were detected in the stomach with palpation. Vigorous attempts to crush the bezoars were unsuccessful; therefore, gastrotomy was performed to retrieve the bezoars. Post hoc anamnesis revealed that the patient had recently ingested a copious amount of Japanese persimmon.

Two patients experienced postoperative morbidity. The first patient developed postoperative ileus four days after the first bezoar operation. Laparotomy revealed a bezoar proximal to the first enterotomy incision. Because its edges were spiked, a segmental resection was performed to retrieve the bezoar. Endto-end intestinal anastomosis was then performed. In fact, the bezoar was observed during the first operation in the stomach, and it was planned to attempt endoscopic digestion after the
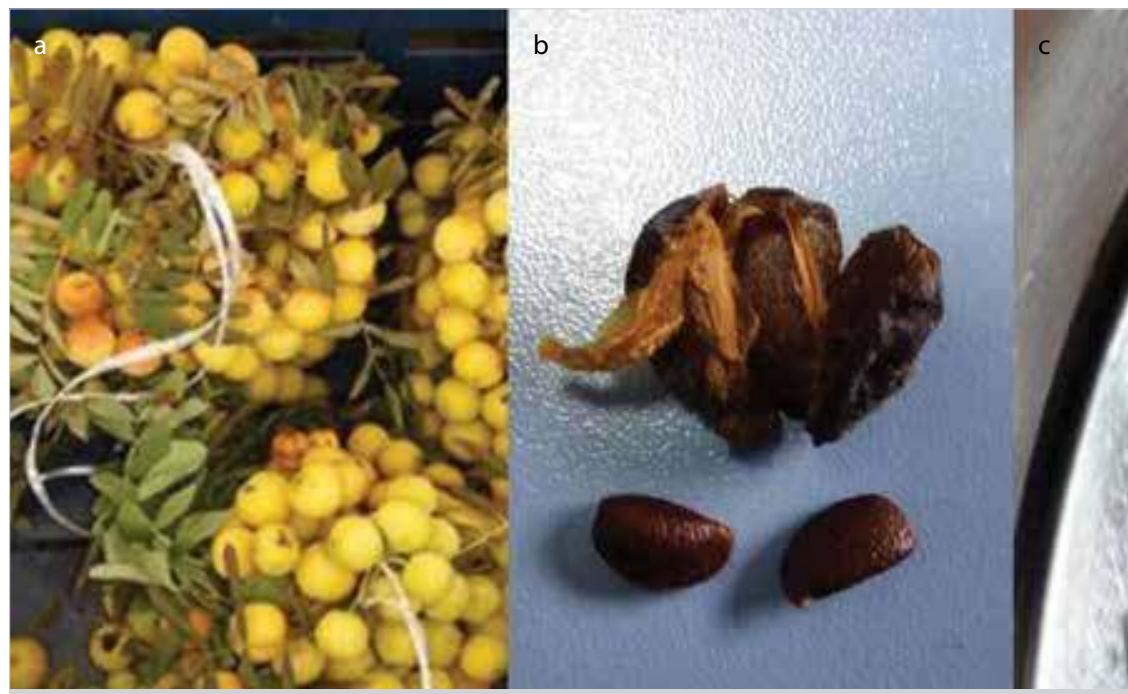

Figure 1. a-c. Sorbus domestica. (a) Fresh SD, (b) dried SD, (c) phytobezoar formation caused by SD

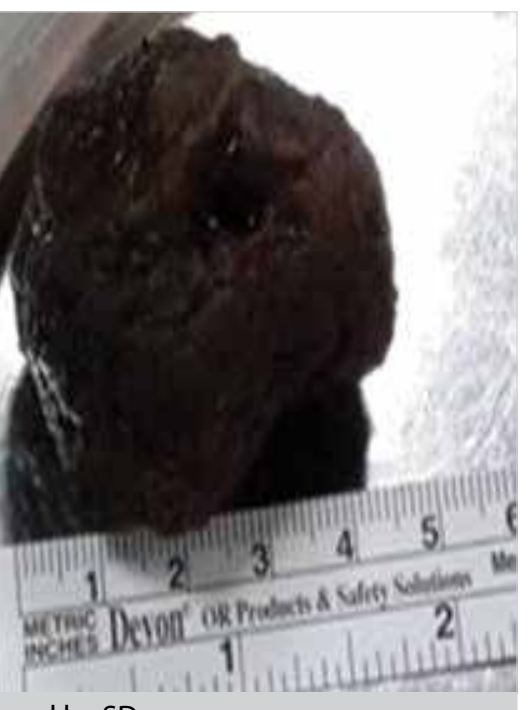


Table 2. Details of clinical management

\begin{tabular}{|c|c|c|c|c|c|c|c|c|c|}
\hline Patients n (\%) & Localization & Diagnosis & S1 & S2 & S3 & S4 & S5 & S6 & $\mathrm{CM}$ \\
\hline $9(45)$ & Small intestine & $\mathrm{CT}$ & 5 & 3 & & & & & 1 \\
\hline $5(25)$ & Stomach & EGD & & & & 1 & & & 4 \\
\hline $5(25)$ & Small intestine and stomach & $E G D, C T$, and during surgery & 1 & & & 1 & 1 & 1 & 1 \\
\hline $1(5)$ & Descending colon & During surgery & & & 1 & & & & \\
\hline
\end{tabular}

S1: Surgery 1 (crushing and passing the contents through the cecum); S2: Surgery 2 (removing the bezoar by enterotomy); S3: Surgery 3 (crushing and passing the contents through the anal canal); S4: Surgery 4 (removing the bezoar by gastrotomy); S5: Surgery 5 (removing the bezoar by gastrotomy and segmental ileal resection); S6: Surgery 6 (removing the bezoar by enterotomy and gastrotomy); CM, conservative management (scattering by endoscopy and ingestion of pineapple juice)

CT: computed tomography; EGD: esophagogastroduodenoscopy
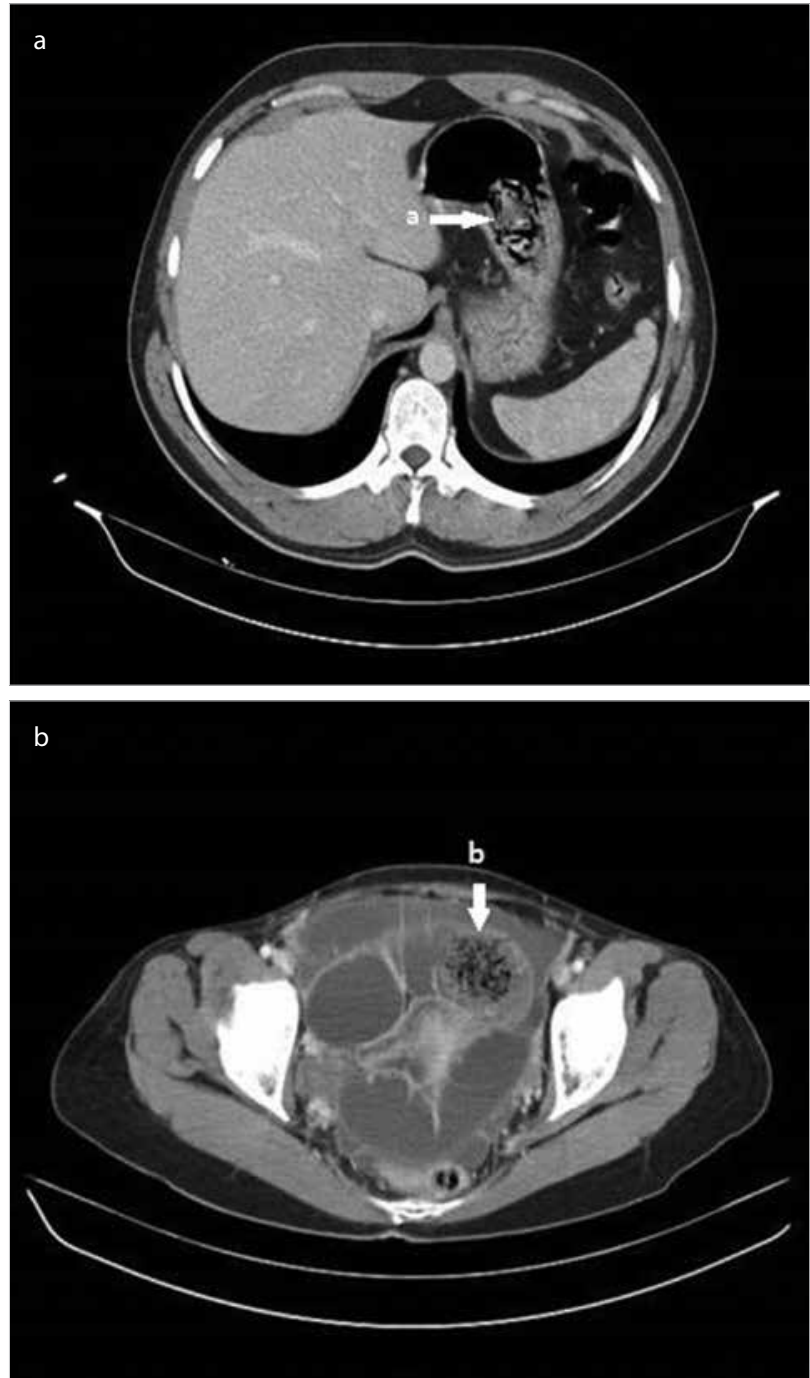

Figure 2. a, b. CT scan showing a phytobezoar. Typical mottled appearance of a phytobezoar in the stomach (a) and distal ileal bowel loops (b)

operation. The second complicatıon was enterocutaneous fistula (treated surgically). There was no mortality. The features of the operations and postoperative periods are presented in Table 3.

\section{DISCUSSION}

Phytobezoars are rare causes of gastrointestinal obstruction. They may arise due to the consumption of fiber-rich and digestive-enzyme-resistant nutrients, such as date palm, citrus
Table 3. Features of the operations and postoperative periods

\begin{tabular}{|lc|}
\hline Minimally invasive surgery & $14(100)$ \\
\hline Conversion to laparotomy & $2(14.2)$ \\
\hline Mean operative time (minutes) & $85.2 \pm 22.1$ \\
\hline Complications & $2(14.2)$ \\
\hline Length of hospital stay (days) & $3.8 \pm 2.4$ \\
\hline Mean follow-up period (months) & $14.3 \pm 14.8$ \\
\hline Morbidity & $2(14.2)$ \\
\hline Mortality & 0 \\
\hline $\mathrm{n}(\%)$ & \\
\hline
\end{tabular}

fruit, quince, fig, coconut, grape, cabbage, and banana (2). Previous gastric surgery, vagotomy-induced hypoacidity, and delay in gastric emptying are believed to be the most important factors facilitating bezoar development; there is a $5 \%$ to $12 \%$ change in the incidence of bezoar after gastrectomy (5-7). Reduced gastric secretion and illnesses such as diabetic neuropathy, hypothyroidism, and myotonic dystrophy associated with motility are additional medical conditions that have been identified as putative predisposing factors (8).

Plants have been used for medicinal purposes for thousands of years. Although the use of herbal medicines showed a decline after the scientific revolution in Europe, it has regained popularity worldwide in recent years. However, the public has many misconceptions related to the medicinal use of herbs. They are considered to be safer than pharmaceutical drugs; furthermore, long-term use at high doses is also accepted as safe. Although many studies have been conducted to assess the adverse effects of herbal drugs and drug-herbal remedy interactions, adverse effects caused by plants that result in the need for surgical intervention have not attracted much attention. SD is a wild fruit that is well known throughout Europe and has been used for medicinal purposes for centuries. It is also used as an adjunct for the treatment of DM. When people with predisposing factors such as diabetic gastropathy and orchronic diseases consume large amounts of the fruit with seeds, they are prone to develop phytobezoars. In our study, 12 of 20 patients recalled the ingestion of copious amounts of SD with seeds. Interestingly, the time between ingestion and the presentation of symptoms was more than two to three months. Therefore, in taking patients' histories of ingestion of 
fruits or vegetables, the period of the history should extend back several months prior to the presentation of symptoms.

Bezoars are often seen in the stomach; however, as they develop, they may not initially have surgical indications. Rather, they may be clinically silent and detected accidentally. When they reach a large size, they may cause gastrointestinal bleeding or perforation. As in some of our cases, the inflammation can be sufficiently severe to be mistaken as malignant ulceration. Bezoars generally cause obstructions in the jejunum or ileum, with signs of intestinal obstruction. If patients have previous surgery in their medical histories, the cases may initially be perceived as ileus, and treatment may be delayed. Abdominal $\mathrm{CT}$ is the most frequently used imaging modality; it has a high success rate in locating obstructions and bezoars. Ripolles et al. (9) stated that the bezoar detection rate using CT imaging is $97 \%$. Bezoars are seen in CT as uniformly bounded, round masses with air bubbles inside and contrast-enhanced edges (10) (Figure 2).

In bezoars in the stomach, upper gastrointestinal system endoscopy can be used both for diagnostic and therapeutic purposes. Gastric phytobezoars are generally treated conservatively. To accomplish this, various methods are conventionally used. Among these, the oldest known method is ingestion of pineapple juice (11). In surgical clinics, repeat doses of cola have been used, and successful outcomes have been reported $(12,13)$. Frequently used proteolytic enzymes for gastric and proximal jejunum bezoars are n-acetylcysteine, papain, and cellulase (14). Gastric bezoars that are nonresponsive to treatment should be scattered using an endoscopic method. In cases with acute abdomen, such as those involving complete intestinal obstruction in the distal gastrointestinal system or perforation, the treatment is surgical. In cases with malnutrition on the intestinal walls, after determining if the partial intestinal resection and intraluminal mass is a bezoar, the passage can be relieved by crushing, splitting, and milking the contents into the cecum (15). Among the surgical treatment options, removing the bezoar via enterotomy is an option; however, enterotomy should only be performed if the bezoar cannot be split or mobilized (16).

Surgical treatment of bezoars is also possible with a laparoscopic approach. Although laparoscopy is difficult to implement for intestinal obstructions, it has been stated that with technical development, laparoscopy can be used safely by experienced physicians and provides better postoperative results $(16,17)$. Yau et al. (6) compared the open approach with the laparoscopic approach in their study of 24 cases of intestinal obstruction due to bezoars; they showed the superiority of laparoscopy in selected patients in whom better postoperative results were obtained. Ganpathi and Cheah (18) identified phytobezoars in the terminal ileum in patients who underwent laparoscopy with a mechanical intestinal obstruction diagnosis and who had no previous history of abdominal surgery; in these patients, the bezoars were extracted using enterotomy. Yol et al. (19) reported that it is also laparoscopically possible to move the bezoar forward into the cecum by crushing it.

As the symptoms of phytobezoars vary greatly, so do the treatment modalities. Observing patients and making several attempts to break down the phytobezoars endoscopically should be the first choice. If the patient presents with acute intestinal obstruction, surgery is inevitable. As can be seen from our study, minimally invasive surgery is feasible and safe in experienced hands. If the mass is movable inside the intestinal lumen, it should be possible to force it into the cecum while taking care not to injure the intestine. However, if the bezoar is stuck or impossible to move without injuring the bowel wall, enterotomy should be performed to remove the phytobezoar. We selected the treatment modality according to the presenting symptoms and clinical conditions of our patients. Another important point during surgery is to control the small bowels and stomach to investigate whether additional bezoars are present. It has been confirmed that $9 \%$ of cases in which operations are performed to remove bezoars undergo a repeat operation (10). To reduce the formation of bezoars and ileus after discharge, exercises should be recommended for patients to develop their mastication functions, and a diet program that is low in fibrous food and high in phytonutrient content should be recommended (20).

\section{CONCLUSION}

In patients with phytobezoar, the treatment modality should be selected for each patient according to the presenting symptoms and characteristics. In the case of surgery, a minimally invasive approach is feasible; ileus can be successfully treated either by crushing and passing the contents through the cecum or by performing enterotomy.

Ethics Committee Approval: Authors declared that the research was conducted according to the principles of the World Medical Association Declaration of Helsinki "Ethical Principles for Medical Research Involving Human Subjects" (amended in October 2013).

Informed Consent: Written informed consent was obtained from patients who participated in this study.

Peer-review: Externally peer-reviewed.

Author Contributions: Concept - Z.Ö., I.O.; Design - Z.Ö., I.O.; Supervision - I..O.; Resource - Z.Ö.; Materials - Z.Ö.; Data Collection and/or Processing - Z.Ö.; Analysis and/or Interpretation - Z.Ö., I.O.; Literature Search - Z.Ö.;Writing Manuscript - Z.Ö.; Critical Reviews - I.O.; Other - Z.Ö.

Conflict of Interest: No conflict of interest was declared by the authors.

Financial Disclosure: The authors declared that this study has received no financial support.

\section{REFERENCES}

1. Ali WA, Gondal ZI, Yammahi AA, Hushki SF, Badri F, El Tayeb YH. A case of small bowel obstruction due to phytobezoars. J Surg Case Rep 2013; pii: rjt046. [CrossRef]

2. Emerson AP. Foods high in fiber and phytobezoar formation. J Am Diet Assoc. 1987; 87: 1675-1677.

3. Tuzlaci E, Aymaz PE. Turkish folk medicinal plants, Part IV: Gönen (Balikesir). Fitoterapia 2001; 72: 323-343. [CrossRef]

4. Termentzi A, Alexiou P, Demopoulos VJ, Kokkalou E. The aldose reductase inhibitory capacity of Sorbus domestica fruit extracts depends on their phenolic content and may be useful for the control of diabetic complications. Pharmazie. 2008; 63: 693-696.

5. Yakan S, Sirinocak A, Telciler KE, Tekeli MT, Deneçli AG. A rare cause of acute abdomen: small bowel obstruction due to phytobezoar. Ulus Travma Acil Cerrahi Derg 2010; 16: 459-463. 
6. Yau KK, Siu WT, Law BK, Cheung HY, Ha JP, Li MK. Laparoscopic approach compared with conventional open approach for bezoarinduced small-bowel obstruction. Arch Surg. 2005; 140: 972-975. [CrossRef]

7. Kement M, Ozlem N, Colak E, Kesmer S, Gezen C, Vural S. Synergistic effect of multiple predisposing risk factors on the development of bezoars. World J Gastroenterol 2012; 18: 960-964. [CrossRef]

8. Teng H, Nawawi O, Ng K, Yik Y. Phytobezoar: an unusual cause of intestinal obstruction. Biomed Imaging Interv J 2005; 1: e4. [CrossRef]

9. Ripollés T, García-Aguayo J, Martínez MJ, Gil P. Gastrointestinal bezoars: sonographic and CT characteristics. AJR Am J Roentgenol 2001; 177: 65-69. [CrossRef]

10. Yildirim T, Yildirim S, Barutcu O, Oguzkurt L, Noyan T. Small bowel obstruction due to phytobezoar: CT diagnosis. Eur Radiol 2002; 12: 2659-2661.

11. Walker-Renard P. Update on the medicinal management of phytobezoars. Am J Gastroenterol 1993; 88: 1663-1666.

12. Ladas SD, Triantafyllou K, Tzathas C, Tassios P, Rokkas T, Raptis SA. Gastric phytobezoars may be treated by nasogastric CocaCola lavage. Eur J Gastroenterol Hepatol. 2002; 14: 801-803. [CrossRef]

13. Ladas S, Kamberoglou D, Karamanolis G, Vlachogiannakos J, Zouboulis-Vafiadis I. Systematic review: Coca-Cola can effectively dissolve gastric phytobezoars as a first-line treatment. Aliment Pharmacol Ther 2013; 37: 169-173. [CrossRef]

14. Gayà J, Barranco L, Llompart A, Reyes J, Obrador A. Persimmon bezoars: a successful combined therapy. Gastrointest Endosc 2002; 55: 581-583. [CrossRef]

15. Naik S, Gupta V, Naik S, Rangole A, Chaudhary AK, Jain P, et al. Rapunzel syndrome reviewed and redefined. Dig Surg. 2007; 24: 157-161. [CrossRef]

16. de Menezes Ettinger JE, Silva Reis JM, de Souza EL, Filho Ede M, Gãlvao do Amaral PC, Ettinger E Jr, et al. Laparoscopic management of intestinal obstruction due to phytobezoar. JSLS 2007; 11: 168-171.

17. Liauw JJ, Cheah Wk. Laparoscopic Management of Acute Small Bowel Obstruction. Asian J Surg 2005; 28: 185-188. [CrossRef]

18. Ganpathi IS, Cheah WK. Laparoscopic-assisted management of small bowel obstruction due to phytobezoar. Surg Laparosc Endosc Percutan Tech 2005; 15: 30-32. [CrossRef]

19. Yol S, Bostanci B, Akoglu M. Laparoscopic treatment of small bowel phytobezoar obstruction. J Laparoendosc Adv Surg Tech A 2003; 13: 325-326. [CrossRef]

20. Parsi S, Rivera C, Vargas J, Silberstein MW. Laparoscopic-assisted extirpation of a phytobezoar causing small bowel obstruction after Roux-en-Y laparoscopic gastric bypass. Am Surg 2013; 79: E93-95. 